\title{
Factors Affecting Uptake of NaF-18 by the Normal Skeleton
}

\author{
Aung Zaw Win ${ }^{\mathrm{a}, \mathrm{c}}$, Carina Mari Aparici ${ }^{\mathrm{b}}$
}

\begin{abstract}
Background: The primary aim of this study was to examine if factors such as renal function, height, weight and age could affect the uptake of sodium fluoride-18 (NaF-18) by the normal bone. This is the first study to examine the possible factors that can influence $\mathrm{NaF}-18$ uptake in the normal bone.
\end{abstract}

Methods: A retrospective study was done on NaF-18 PET/CT bone scans from January 2010 to May 2012 at our institution. All NaF-18 PET/CT studies used the same clinical protocol. Our excluding criteria were patients with abnormal renal function and patients with past history of cancer and metabolic bone diseases. Spearman's correlation was used to analyze the data.

Results: From our study ( $\mathrm{n}=11$ patients), no correlation was found between $\mathrm{SUV}_{\text {max }}$ and serum creatinine and between $\mathrm{SUV}_{\text {max }}$ and age. However, significant correlations were found between $\mathrm{SUV}_{\max }$ and height $(\mathrm{cm})$ and between $\mathrm{SUV}_{\text {max }}$ and weight $(\mathrm{kg})$ for thoracic $5,7,12$ and lumbar 2 vertebral levels.

Conclusion: Based on our findings, $\mathrm{SUV}_{\text {max }}$ values in NaF-18 PET/ CT bone scans can vary depending on the patient's height, weight and bone region. This information can be helpful in diagnosing and monitoring bone pathologies and can help explain the clinical findings.

Keywords: SUV; Bone; NaF-18 PET/CT; Bone scan

Manuscript accepted for publication July 31, 2014

${ }^{\mathrm{a}}$ Department of Radiology, San Francisco VA Medical Center, 4150 Clement Street, San Francisco, CA 94121, USA

${ }^{\mathrm{b}}$ Department of Radiology, University California San Francisco (UCSF), 500 Parnassus Ave, San Francisco, CA 94143, USA

${ }^{\mathrm{c}}$ Corresponding Author: Aung Zaw Win, Department of Radiology, San Francisco VA Medical Center, 4150 Clement Street, San Francisco, CA 94121, USA. Email: aungzwin@gmail.com

doi: http://dx.doi.org/10.14740/jocmr1901w

\section{Introduction}

SUV value is defined as the tissue concentration of tracer as measured by a PET scanner divided by the activity injected divided by body weight [1]. The uptake value is represented by pixel or voxel intensity value in the region of interest (ROI) of the image, which is then converted into the activity concentration. Previous studies on sodium fluoride-18 (NaF18) PET/CT have reported correlations between SUV and lean body mass, and between SUV and total body weight [2-4]. However, SUV values vary depending on the organ of the body. SUV of bone depends on the blood flow to the area, exposed bone surface area, regional osteoblastic activity and on renal clearance [5]. The tracer uptake in bone does not plateau for several hours [1]. NaF-18 is excreted by the kidneys. Age-related changes of the bone are more pronounced in some locations compared to others. For example, parietal and occipital bones show more age-related changes compared to the rest of the skull [6].

The ability to reliably follow changes in SUV can be used to assess the changes due to therapy in cancer cases. It must be noted that blood flow varies among different bones [7]. The diffusion of NaF into the bone leads to a slow exchange of fluoride ions with hydroxide ions of the hydroxyapatite crystals, eventually forming fluoroapatite, a process that begins rapidly but takes many days to weeks to complete [5]. The rapid uptake of $18 \mathrm{~F}$-fluoride occurs preferentially at sites of high osteoblastic activity where bone remodeling is greatest. Hence the component of bone turnover being measured by $18 \mathrm{~F}$ imaging is osteoblastic activity [8]. Yet, the tracer can accumulate in both osteoblastic and osteolytic lesions [9]. F18 ion has a high affinity for bone that leads to a large tissue to background ratio and hence good-quality images [10]. NaF-18 has two-fold greater tracer accumulation in skeletal system compared with Tc99m-MDP [11]. Unlike Tc $99 \mathrm{~m}$ tracer, there is no protein binding for NaF-18 and NaF18 has faster blood and renal clearance [12]. NaF-18 PET/ $\mathrm{CT}$ bone scan has less radiation exposure than Tc99m-MDP SPECT/CT [13]. Moreover, Tc99m-MDP tracer requires a delay of $2 \mathrm{~h}$ or greater before imaging can be performed [14]. The usual imaging time is $45-60 \mathrm{~min}$ for NaF-18 PET 
Table 1. Patient Characteristics

\begin{tabular}{lll}
\hline Patient & Age & Reason for NaF-18 PET/CT bone scan \\
\hline 1 & 54 & Abnormal right femur, to rule out metastasis \\
2 & 50 & Assess right seventh rib incidental finding \\
3 & 66 & Evaluate for lytic lesions found on CT \\
4 & 62 & Presumed metastasis to bones \\
5 & 89 & Sclerotic lesion on X-ray \\
6 & 42 & To rule out skeletal coccidioidomycosis \\
7 & 63 & Status post left total hip arthroplasty with bone pain \\
8 & 65 & Knee joint replacement \\
9 & 62 & Arthropathy of left ankle \\
10 & 83 & Benign prostatic hyperplasia (BPH) \\
11 & 77 & Elevated PSA \\
\hline
\end{tabular}

studies. Even-Sapir et al reported that NaF-18 PET/CT has a sensitivity and specificity of $100 \%$ respectively for detecting prostate cancer metastases [15]. It is certainly more sensitive and more specific than Tc99m-MDP bone scan.

NaF-18 PET/CT can detect skeletal metastases of tumors that typically have low FDG avidity, such as thyroid cancer or renal cell cancer [16]. A study by Nakai et al found that FDG PET had a low visualization rate in cases of osteoblastic bone metastases [17]. Moreover, NaF-18 can accurately differentiate between benign and malignant lesions [18]. No limitations to diet or physical activity are required for this exam, whereas, for FDG PET/CT, the patient has to fast overnight and limit physical activity to avoid increased FDG uptake by the muscles. For bone imaging, there is high uptake of FDG tracer by bone marrow, whereas with NaF18, uptake of tracer by the bone marrow is negligible [7].

Increased NaF-18 accumulation is observed in inflammations, past trauma, fibrous dysplasia, Paget's disease, hyperostosis frontalis, and myositis ossificans [11]. Quantitative 18F-fluoride PET may prove useful for the assessment of metabolic bone disorders such as renal osteodystrophy, osteoporosis, or Paget's disease [19]. It has been used to assess metabolic, degenerative, traumatic, and neoplastic bone diseases. Many studies have been done on patients with osteoporosis and patients with bone metastasis. Cancer and metabolic bone diseases such as osteoporosis, osteopenia and Paget's disease can alter the SUV values. Kurdziel et al carried out a prospective study with multiple myeloma and prostate cancer patients and they calculated SUV from NaF18 PET/CT exams [5]. Research on SUV in NaF-18 exams is very limited. The primary aim of this study was to examine if factors such as renal function, height, weight and age could affect the uptake of sNaF-18 by the normal bone. This is the first study to examine the possible factors that can influence NaF-18 uptake in the normal bone.

\section{Methods}

We retrospectively reviewed all the NaF-18 PET/CT bone scans done at our institution between January 1, 2010 and May 31, 2012. All NaF-18 PET/CT studies used the same clinical protocol. We excluded patients with histories of cancer and metabolic bone diseases such as osteopororsis, osteopenia and Paget's disease. We included patients with degenerative changes in bone, patients who had arthroplastic surgery and patients with prosthetic devices in bone. We collected information on age, gender, serum creatinine values, height $(\mathrm{cm})$, and weight $(\mathrm{kg})$. The patients in this study (n $=11$ ) had normal renal function based on the serum creatinine values. Two nuclear medicine physicians independently measured $\mathrm{SUV}_{\text {mean }}$ values in 31 locations (C1-C7, T1-T12, L1-L5, right and left femoral head, right and left humeral head, mid sternum on the level of T3, and right and left parietal bones) on the axial and appendicular skeletons. Areas with degenerative changes were avoided. The ROI used in 

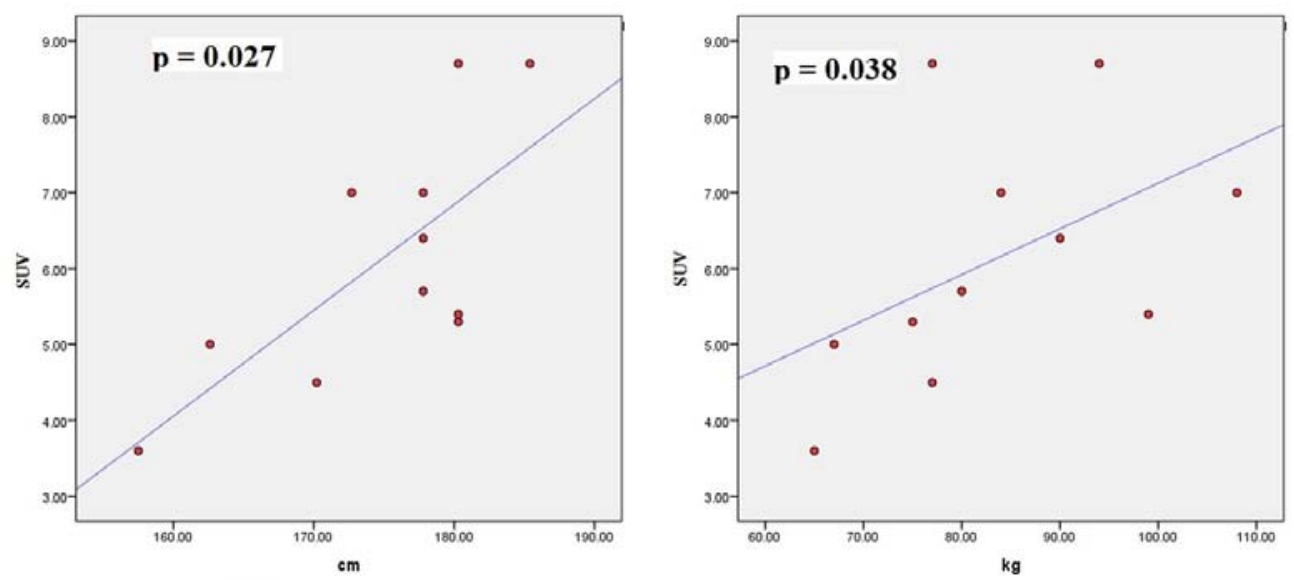

T5 vertebra
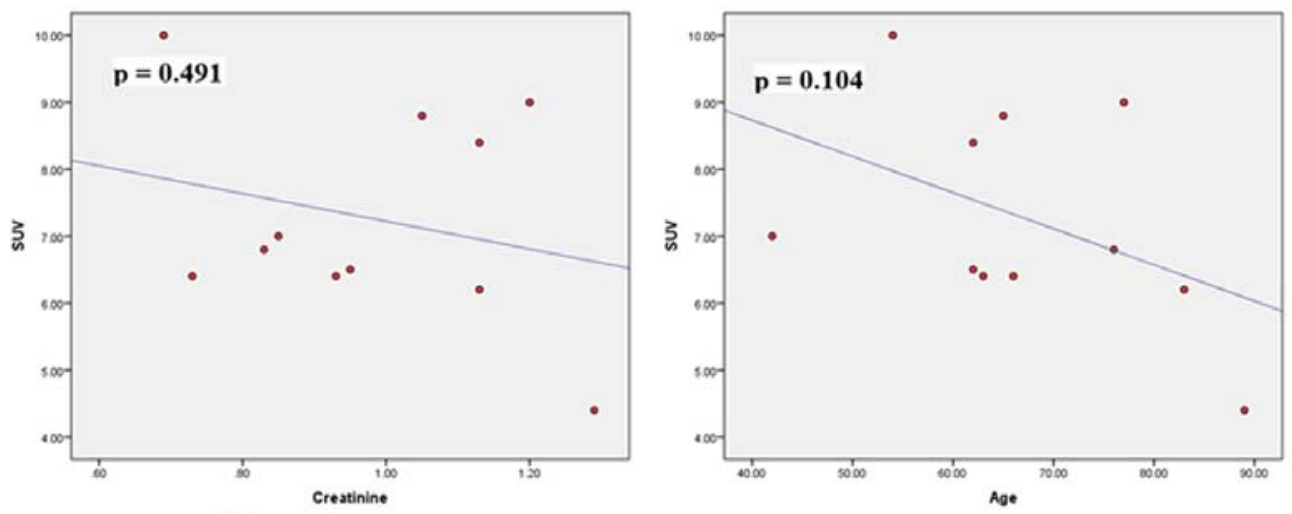

T5 vertebra

Figure 1. Scatter plots correlating SUV ${ }_{\max }$ of $T 5$ with height $(\mathrm{cm})$, weight $(\mathrm{kg})$, creatinine, and age (years).

this study was $826 \mathrm{~mm}^{3}$.

\section{Technique}

Sixty minutes following the intravenous administration of NaF-18, CT transmission images without intravenous contrast were acquired from the vertex to the toes for attenuation correction and anatomic localization. This was followed by a PET scan over the same anatomical regions. A rotating 3D MIP, as well as axial, coronal and sagittal PET images with and without attenuation correction was interpreted. Acquired CT and PET/CT images were reviewed alongside the PET images. The dose of NaF-18 injected ranged from 9.5 to $12 \mathrm{mCi}$. Approximately $1 \mathrm{~h}$ after injection with the isotope, imaging was performed in a PET/CT scanner (GE STE 64 slice CT scanner, GE Healthcare, Waukesha, WI, USA) with a CT imaging system. CT scan was done for attenuation correction and anatomic localization. Imaging parameters were $140 \mathrm{kVp}, 90-120 \mathrm{~mA}, 1.25 \mathrm{~mm}$ collimation with reconstruction as $3.75 \mathrm{~mm}$ thick sections by using a $512 \times 512$ matrix and a filtered back projection algorithm. Immediately after CT, PET images were obtained in two-dimensional mode for 4-min acquisitions at each level for one bed position. PET images were reconstructed by using a $128 \times 128$ matrix and an iterative-ordered subset expectation maximization algorithm.

\section{Statistics}

We used the SPSS version 20 to analyze the data. Spearman's correlation was used and a $\mathrm{P}$ value of less than 0.05 was considered significant for this study.

\section{Results}

The characteristics of patients are shown in Table 1. The average normal $\mathrm{SUV}_{\text {max }}$ values from 11 patients were cervical vertebrae 6.84 , thoracic vertebrae 7.36 , lumbar vertebrae 7.27, femoral head 2.22, humeral head 1.82, mid sternum 5.51 , and parietal bone 1.71 . The mean age of the patients was 64.8 years and ranged from 42 to 89 . The serum creati- 

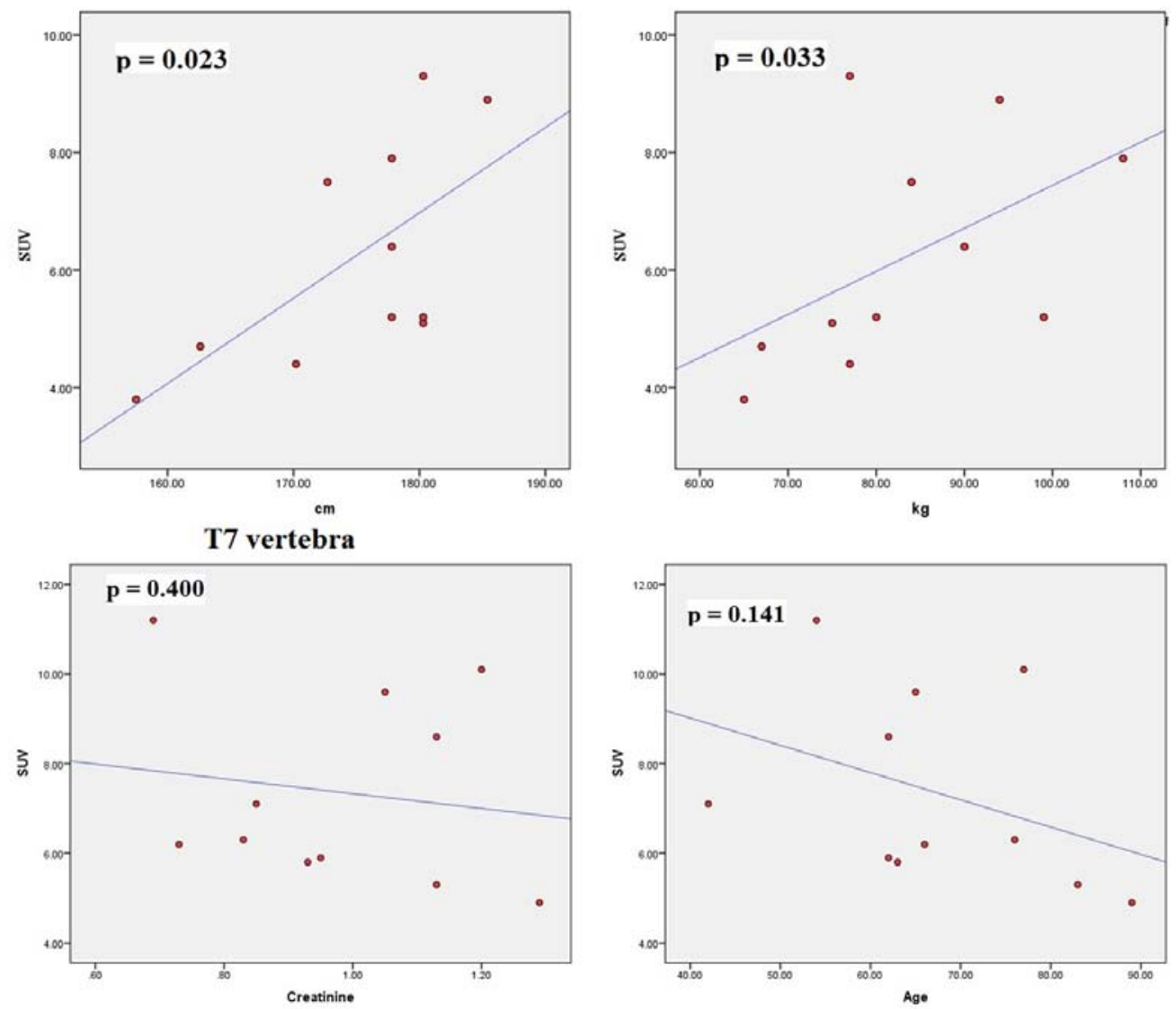

T7 vertebra

Figure 2. Scatter plots correlating SUV $\mathrm{max}_{\text {ax }}$ of $\mathrm{T7}$ with height $(\mathrm{cm})$, weight $(\mathrm{kg})$, creatinine, and age (years).

nine levels ranged from 0.64 to 1.29 (normal $0.6-1.3$ ). The mean height was $174.79 \mathrm{~cm}$ (range $157.5-185.4$ ) and the mean body weight was $83.27 \mathrm{~kg}$ (range 65 - 108). The dose of NaF-18 injected ranged from 9.5 to $12.6 \mathrm{mCi}$ depending on the weight of the patient. We found no significant correlation between SUV and serum creatinine. There were also no significant correlations between SUV and age. However, significant correlations were found between $\mathrm{SUV}_{\max }$ and height $(\mathrm{cm})$ and between $\mathrm{SUV}_{\text {max }}$ and weight $(\mathrm{kg})$ for thoracic 5, 7, 12 and lumbar 2 vertebral levels.

The scatter plots of linear regression lines are shown in Figures 1-4.

\section{Discussion}

NaF-18 tracer is eliminated from the body by the kidneys. However, we did not find a significant correlation between SUV and serum creatinine. One possible explanation may be that we would start to see a significant correlation once the serum creatinine value is above the normal range. All of our patients had serum creatinine values within the nor- mal range and within that range, changes in SUV due to changes in creatinine level may be minimal. Another reason may be that nine out $11(82 \%)$ patients in this study were overweight or obese. Creatinine production rate is decreased with reduced lean body mass [20]. Creatinine is formed in muscle and it is proportional to muscle mass. Older patients have reduced muscle mass. Moreover, commonly used drugs such as aspirin can have an effect on creatinine levels. From our study, age does not seem to affect the SUV uptake by bone. The mean age of our patients with past histories of arthralgia, joint replacement and osteoarthritis was 58.8 years, compared to the mean age of 64.8 years for the whole study population. As a result SUV uptake in younger patients may be high compared to older patients in this study. This might contribute to the fact that we found no significant correlation between age and SUV.

Osteoblastic activity decreases with age. So, it is not surprising that we observed a negative trend for age in all four bone levels shown in the graphs. Serum creatinine reflects kidney function and glomerular filtration rate (GFR). Renal fluoride clearance is related to the GFR, and NaF-18 PET/ $\mathrm{CT}$ was used to assess renal function in rats [21]. Fluoride 

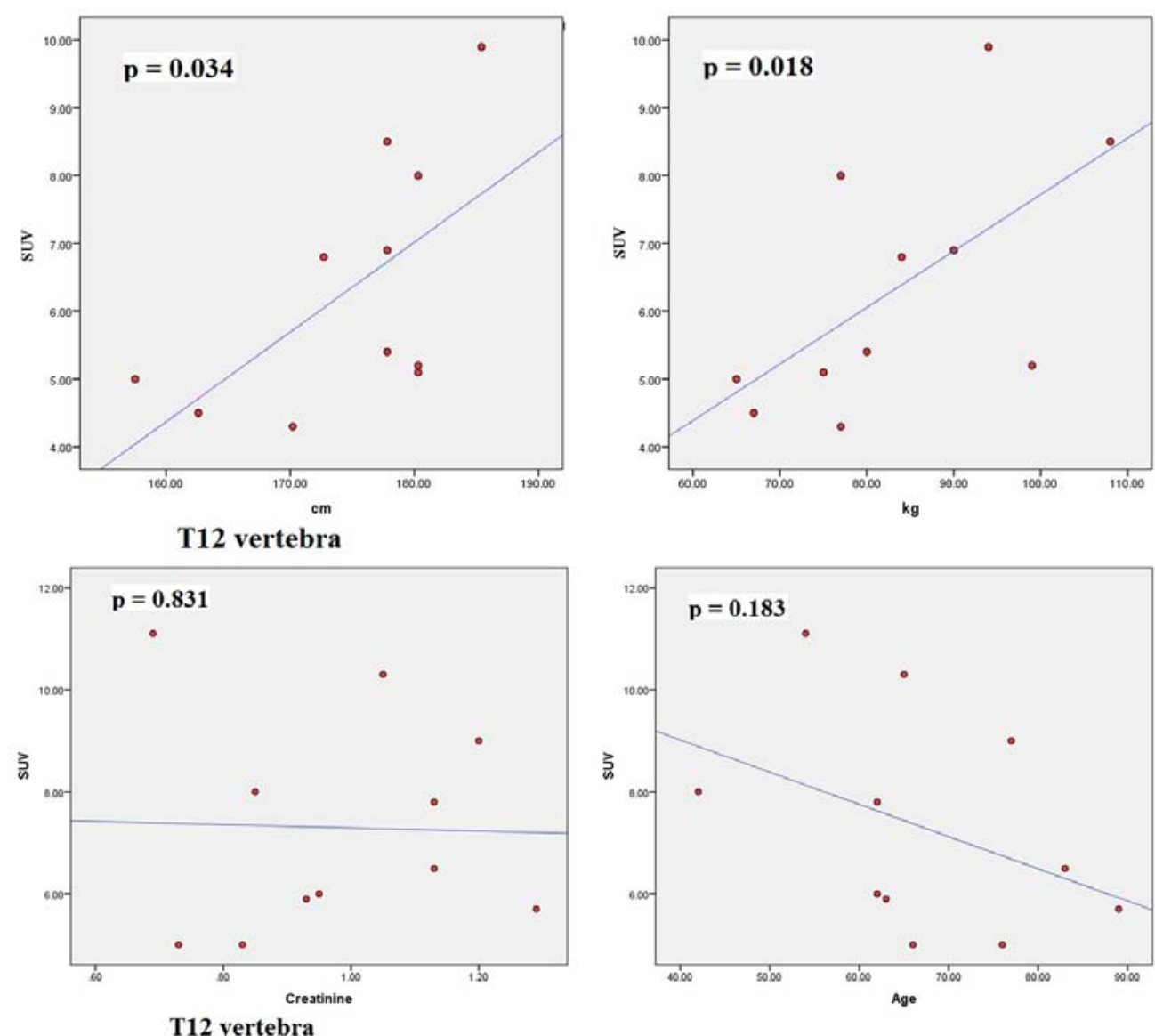

Figure 3. Scatter plots correlating $\mathrm{SUV}_{\max }$ of $\mathrm{T} 12$ with height $(\mathrm{cm})$, weight $(\mathrm{kg})$, creatinine, and age (years).

ions are freely filtered in the glomeruli but they also undergo tubular reabsorption [22]. Tubular reabsorption of fluoride increases with decreasing GFR [22]. Therefore, a negative relationship is expected between fluoride ions (SUV) and creatinine. This agrees with our results. However, for both age and creatinine, we did not see a statistically significant association with SUV, even though a negative relationship existed.

There were significant associations between $\mathrm{SUV}_{\max }$ and height $(\mathrm{cm})$ and between $\mathrm{SUV}_{\text {max }}$ and weight $(\mathrm{kg})$ for thoracic 5, 7, 12 and lumbar 2 vertebral levels. Increased weight puts mechanical stress on the spine. Mechanical stress enhances interleukin 11 expression and this stimulates osteoblast differentiation [23]. Increased osteoblastic activity is associated with high SUV uptake. It can be further assumed that in obese persons, there is a significant risk of osteoarthritis developing in thoracic and lumbar vertebra, particularly at T5, T7, T12 and L2 levels. Borenstein reported that the lumbar spine is a common location for osteoarthritis [24]. O'Neill et al studied 681 women and 499 men with osteoarthritis and they found that obesity (increased weight) was associated with osteoarthritis at L3 vertebra [25]. In our study, increased tracer uptake in L2 was associated with increased weight.

In the vertebral column, thoracic and especially the lumbar vertebra bear the majority of the body weight and they absorb the stress of lifting heavy objects [26]. As a result, lumbar spine has significant bone turnover [27] and this may be part of the reason why there was correlation between SUV of lumbar spine and height and weight. Suenaga et al measured the SUV of mandibular bone using NaF-18 PET/CT [28]. They find that by using this method, they can detect mechanobiological changes in bone due to bone turnover from wear. Another reason for the association between SUV and body weight in NaF-18 bone studies may be that weight causes changes in geometry and structure on certain bones in the body [26]. A correlation between SUV and total body weight was reported in FDG PET studies [2-4].

Keyes reported that PET images should be interpreted subjectively rather than quantitative interpretation of SUV [1]. In contrast, Cook et al reported that SUV has predictive value for evaluating bone metastases, whereas, qualitative (visual) interpretation tended to be less valuable [29]. SUV measurements from NaF-18 PET/CT have the potential to 

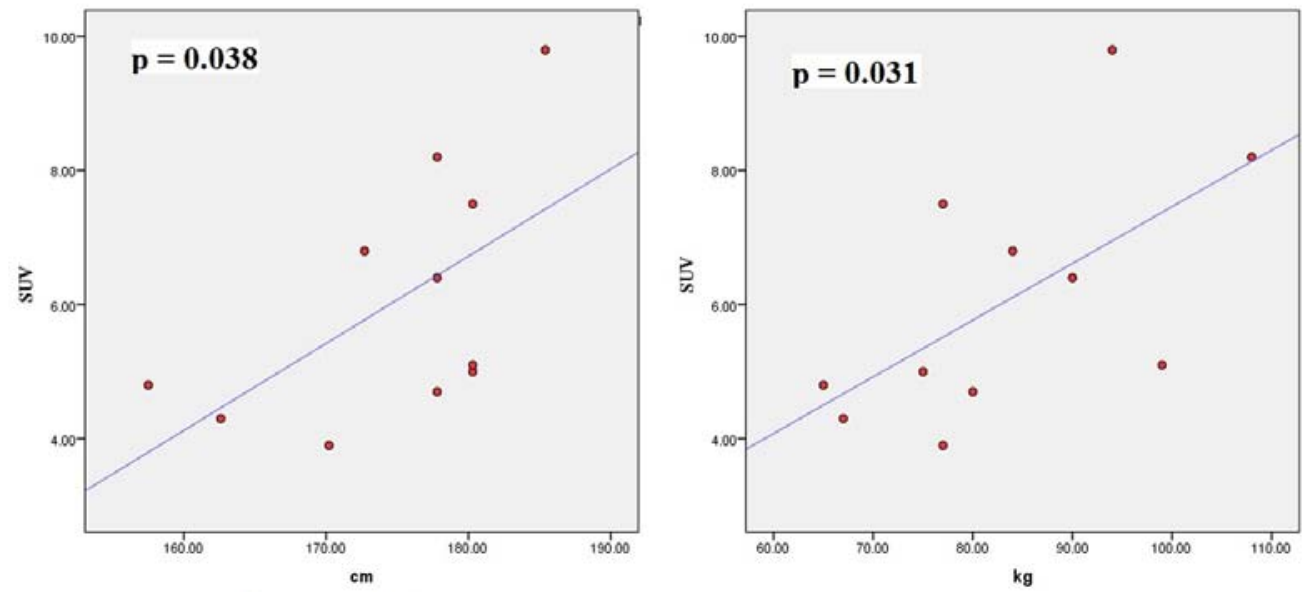

L2 vertebra
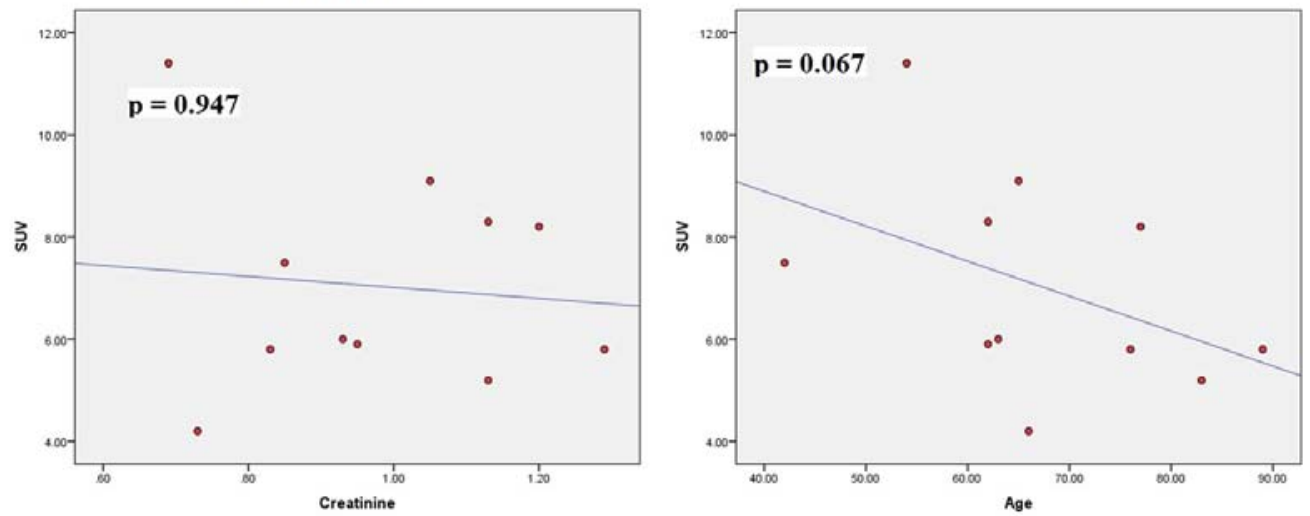

L2 vertebra

Figure 4. Scatter plots correlating $\mathrm{SUV}_{\max }$ of L2 with height $(\mathrm{cm})$, weight $(\mathrm{kg})$, creatinine, and age (years).

monitor treatment response [22]. In patients with osteoporosis, SUV obtained from NaF-18 PET/CT studies significantly decreased after treatment with bisphosphonates [30]. This shows that simple SUV measurements may be sufficient for monitoring disease response in metabolic bone diseases. Simplified SUV uptake measurements may be suitable substitutes for more complex kinetic modeling [22].

Cancellous bone is less dense than cortical bone. It typically occupies the interior region of bones, and is highly vascular [3]. Thus, cancellous bone can have more SUV uptake than cortical bone. Cortical bone forms most of the bone mass, but it represents the minority of bone surface [22]. By comparison, cancellous bone forms only $20 \%$ of the bone mass but accounts for $80 \%$ of the bone turnover associated with remodeling [22]. The spine is rich in cancellous bone and it is the best site for quantitative assessment of bone metabolism because bone turnover is greater than that observed at other skeletal sites [31]. Lastly, unlike the prospective study of NaF-18 by Kurdziel et al, this is a retrospective study and the dosage of NaF-18 in our studies was the same as that used in routine exams [5]. Thus, our results are com- parable to other routine exams.

\section{Conclusion}

Many factors such as age, lean body mass, height, gender, and weight can possibly influence SUV values in NaF PET/CT imaging. Based on our findings, $\mathrm{SUV}_{\text {max }}$ values in NaF-18 PET/CT bone scans can vary depending on the patient's height, weight and bone region. NaF-18 tracer is eliminated from the body by the kidneys; however, we did not find a significant correlation between SUV and serum creatinine. Simplified measurements of SUV on NaF-18 PET/CT promise to improve clinical application. This information can be helpful in diagnosing and monitoring bone pathologies and can help explain the clinical findings. Further studies involving multiple institutions and larger sample sizes can be done to further confirm our findings.

\section{Acknowledgement}

None. 


\section{Conflict of Interest}

Authors declare that they have no conflict of interests.

\section{Funding}

No funding was received for this work.

\section{References}

1. Keyes JW, Jr. SUV: standard uptake or silly useless value? J Nucl Med. 1995;36(10):1836-1839.

2. Sugawara Y, Zasadny KR, Neuhoff AW, Wahl RL. Reevaluation of the standardized uptake value for FDG: variations with body weight and methods for correction. Radiology. 1999;213(2):521-525.

3. Schomburg A, Bender H, Reichel C, Sommer T, Ruhlmann J, Kozak B, Biersack HJ. Standardized uptake values of fluorine- 18 fluorodeoxyglucose: the value of different normalization procedures. Eur J Nucl Med. 1996;23(5):571-574.

4. Zasadny KR, Wahl RL. Standardized uptake values of normal tissues at PET with 2-[fluorine-18]-fluoro-2-deoxy-D-glucose: variations with body weight and a method for correction. Radiology. 1993;189(3):847-850.

5. Kurdziel KA, Shih JH, Apolo AB, Lindenberg L, Mena E, McKinney YY, Adler SS, et al. The kinetics and reproducibility of $18 \mathrm{~F}$-sodium fluoride for oncology using current PET camera technology. J Nucl Med. 2012;53(8):1175-1184.

6. Laurent V, Trausch G, Bruot O, Olivier P, Felblinger J, Regent D. Comparative study of two whole-body imaging techniques in the case of melanoma metastases: advantages of multi-contrast MRI examination including a diffusion-weighted sequence in comparison with PET-CT. Eur J Radiol. 2010;75(3):376-383.

7. Cheng G. Using a cut-off SUV level to define bone marrow lesions on FDG PET is not appropriate. Ann Hematol. 2013;92(2):283-284.

8. Frost ML, Siddique M, Blake GM, Moore AE, Schleyer PJ, Dunn JT, Somer EJ, et al. Differential effects of teriparatide on regional bone formation using (18)F-fluoride positron emission tomography. J Bone Miner Res. 2011;26(5):1002-1011.

9. Sachpekidis C, Goldschmidt H, Hose D, Pan L, Cheng C, Kopka K, Haberkorn U, et al. PET/CT studies of multiple myeloma using (18) F-FDG and (18) F-NaF: comparison of distribution patterns and tracers' pharmacokinetics. Eur J Nucl Med Mol Imaging. 2014;41(7):1343-1353.

10. Al-Beyatti Y, Siddique M, Frost ML, Fogelman I, Blake
GM. Precision of (1)(8)F-fluoride PET skeletal kinetic studies in the assessment of bone metabolism. Osteoporos Int. 2012;23(10):2535-2541.

11. Siddique M, Blake GM, Frost ML, Moore AE, Puri T, Marsden PK, Fogelman I. Estimation of regional bone metabolism from whole-body $18 \mathrm{~F}$-fluoride PET static images. Eur J Nucl Med Mol Imaging. 2012;39(2):337343.

12. Blake GM, Siddique M, Frost ML, Moore AE, Fogelman I. Radionuclide studies of bone metabolism: do bone uptake and bone plasma clearance provide equivalent measurements of bone turnover? Bone. 2011;49(3):537-542.

13. Freudenberg R, Oehme L, Kotzerke J. Radiation exposure should not limit bone scintigraphy with $18 \mathrm{~F}-\mathrm{NaF}$. J Nucl Med. 2012;53(11):1817-1818; author reply 18181819.

14. Liu Y. Setting an SUV cut-off value is misleading and meaningless in the differentiation between physiological and pathological accumulations in the head and neck. Nucl Med Commun. 2009;30(11):895; author reply 895896.

15. Even-Sapir E, Metser U, Mishani E, Lievshitz G, Lerman H, Leibovitch I. The detection of bone metastases in patients with high-risk prostate cancer: $99 \mathrm{mTc}-\mathrm{MDP}$ Planar bone scintigraphy, single- and multi-field-ofview SPECT, 18F-fluoride PET, and 18F-fluoride PET/ CT. J Nucl Med. 2006;47(2):287-297.

16. Grant FD, Fahey FH, Packard AB, Davis RT, Alavi A, Treves ST. Skeletal PET with 18F-fluoride: applying new technology to an old tracer. J Nucl Med. 2008;49(1):6878.

17. Nakai T, Okuyama C, Kubota T, Yamada K, Ushijima Y, Taniike K, Suzuki T, et al. Pitfalls of FDG-PET for the diagnosis of osteoblastic bone metastases in patients with breast cancer. Eur J Nucl Med Mol Imaging. 2005;32(11):1253-1258.

18. Schirrmeister H, Guhlmann A, Elsner K, Kotzerke J, Glatting G, Rentschler M, Neumaier B, et al. Sensitivity in detecting osseous lesions depends on anatomic localization: planar bone scintigraphy versus $18 \mathrm{~F} \mathrm{PET}$. J Nucl Med. 1999;40(10):1623-1629.

19. Fogelman I, Cook G, Israel O, Van der Wall H. Positron emission tomography and bone metastases. Semin Nucl Med. 2005;35(2):135-142.

20. Wu I, Parikh CR. Screening for kidney diseases: older measures versus novel biomarkers. Clin J Am Soc Nephrol. 2008;3(6):1895-1901.

21. Schnockel U, Reuter S, Stegger L, Schlatter E, Schafers KP, Hermann S, Schober O, et al. Dynamic 18F-fluoride small animal PET to noninvasively assess renal function in rats. Eur J Nucl Med Mol Imaging. 2008;35(12):22672274.

22. Wong KK, Piert M. Dynamic bone imaging with $99 \mathrm{mTc}-$ labeled diphosphonates and 18F-NaF: mechanisms and 
applications. J Nucl Med. 2013;54(4):590-599.

23. Kobayashi N, Inaba Y, Tateishi U, Yukizawa Y, Ike H, Inoue T, Saito T. New application of 18F-fluoride PET for the detection of bone remodeling in early-stage osteoarthritis of the hip. Clin Nucl Med. 2013;38(10):e379-383.

24. Borenstein D. Does osteoarthritis of the lumbar spine cause chronic low back pain? Curr Rheumatol Rep. 2004;6(1):14-19.

25. O’Neill TW, McCloskey EV, Kanis JA, Bhalla AK, Reeve J, Reid DM, Todd C, et al. The distribution, determinants, and clinical correlates of vertebral osteophytosis: a population based survey. J Rheumatol. 1999;26(4):842-848.

26. Singer K, Edmondston S, Day R, Breidahl P, Price R. Prediction of thoracic and lumbar vertebral body compressive strength: correlations with bone mineral density and vertebral region. Bone. 1995;17(2):167-174.

27. Frost ML, Cook GJ, Blake GM, Marsden PK, Fogelman I. The relationship between regional bone turnover measured using 18F-fluoride positron emission tomography and changes in BMD is equivalent to that seen for biochemical markers of bone turnover. J Clin Densitom.
2007;10(1):46-54.

28. Suenaga H, Yokoyama M, Yamaguchi K, Sasaki K. Bone metabolism of residual ridge beneath the denture base of an RPD observed using NaF-PET/CT. J Prosthodont Res. 2012;56(1):42-46.

29. Cook G, Jr., Parker C, Chua S, Johnson B, Aksnes AK, Lewington VJ. 18F-fluoride PET: changes in uptake as a method to assess response in bone metastases from castrate-resistant prostate cancer patients treated with 223Ra-chloride (Alpharadin). EJNMMI Res. 2011;1(1):4.

30. Installe J, Nzeusseu A, Bol A, Depresseux G, Devogelaer JP, Lonneux M. (18)F-fluoride PET for monitoring therapeutic response in Paget's disease of bone. J Nucl Med. 2005;46(10):1650-1658.

31. Cheng C, Heiss C, Dimitrakopoulou-Strauss A, Govindarajan P, Schlewitz G, Pan L, Schnettler R, et al. Evaluation of bone remodeling with (18)F-fluoride and correlation with the glucose metabolism measured by (18) F-FDG in lumbar spine with time in an experimental nude rat model with osteoporosis using dynamic PETCT. Am J Nucl Med Mol Imaging. 2013;3(2):118-128. 